\title{
LINKING THE PROPERTIES, PROCESSING AND CHEMISTRY OF ADVANCED SINGLE CRYSTAL NI-BASE SUPERALLOYS
}

\author{
S. $\operatorname{Tin}^{1}$, L. Zhang ${ }^{2}$, R.A. Hobbs ${ }^{3}$, A.-C. Yeh², C.M.F. Rae ${ }^{2}$ and B. Broomfield ${ }^{3}$ \\ ${ }^{1}$ Illinois Institute of Technology, 10 W. $32^{\text {nd }}$ St. Chicago IL 60616 \\ ${ }^{2}$ University of Cambridge, Pembroke Street, Cambridge, CB2 3QZ, UK \\ ${ }^{3}$ Rolls-Royce plc, PO Box 31, Derby DE24 8BJ, UK
}

Keywords: Ruthenium, superalloy, single crystal, creep and fatigue

\begin{abstract}
As nickel-base single crystals are being implemented in gas turbine engines operating at temperatures in excess of $1600 \mathrm{~K}$, maintaining structural properties at these elevated temperatures has become an increasingly important problem. Typically, elevated levels refractory alloying elements are added to these single crystal alloys to enhance the degree of solid solution strengthening within the microstructure. The presence of Re, W, Mo, Ta in Ni-base superalloys strongly influences parameters, such as the stacking fault energies, lattice misfit, and shear modulus of the crystalline lattices, that govern the creep response of the material. However, this is a challenge when dealing with high refractory alloys that are susceptible to the precipitation of deleterious topologically-close-packed (TCP) phases. Recent investigations have demonstrated $\mathrm{Ru}$ additions to be beneficial with respect to hindering the formation of these TCP phases and consequently improving the creep properties of these advanced single crystal alloys. Substantial effort has been dedicated towards understanding the fundamental effects and the mechanisms by which Ru additions alter the structure of the $\gamma$ and $\gamma^{\prime}$ phases. Much of this knowledge has been effectively applied to the development of a new class of single crystal Ni-base superalloys with improved strength and a temperature capability significantly higher than those of existing "second" or "third" generation alloys. Nevertheless, optimization of the alloy chemistries remains notoriously difficult as various component design requirements related to the solidification characteristics, overall blade density, coating compatibility and cost must also be carefully considered when developing these materials. This investigation addresses some of the issues associated with the development of Ru-bearing, high refractory content Ni-base superalloys and describes how interrelationships between the properties, processing, microstructure and chemistry of these alloys can be balanced to yield a practical "fourth" generation single crystal superalloy.
\end{abstract}

\begin{abstract}
Introduction
Nickel-base superalloys are part of an extraordinary class of structural materials that are critical to the continued development of high performance turbine engines for marine transport, aerospace propulsion and power generation. Environmental regulations restricting levels of $\mathrm{CO}_{2}$ output combined with escalating fuel and oil costs has triggered great interest in the development of ultra-efficient turbines, such as those used on the new Boeing 787 Dreamliner and Airbus A350 civil transport jetliners. The ability to operate turbines at higher temperatures is key in minimizing the specific fuel consumption (SFC) and increasing the overall thermodynamic efficiency of engine cycle.
\end{abstract}

During operation, static and rotating structural components located in the hottest section of the turbine engine are required to sustain large mechanical loads while being exposed to corrosive high temperature environments. Turbine blades and nozzle guide vanes in the high-pressure stage of the engine are the primary structural components that are exposed directly to the combustion gases whose temperature may be in excess of $1450^{\circ} \mathrm{C}$. Despite the use of complex internal cooling systems and ceramic thermal barrier coatings, the substrate must be able to tolerate temperatures of $1150^{\circ} \mathrm{C}$ with occasional excursions (or local hot spots) to temperatures as high as $1200^{\circ} \mathrm{C}^{[1]}$. For the past two decades, single crystal Ni-base superalloys have been used extensively in these types of applications where extreme resistance to high temperature deformation is required. As a result, a variety of compositional and microstructural changes have been utilized to enhance the mechanical properties of this class of alloys. The compositions of Ni-base single crystal superalloys have been gradually modified throughout the years with increasing levels of refractory alloying additions to improve creep properties and extend the temperature capability of the alloy ${ }^{[2-6]}$. These compositional changes combined with carefully controlled heat treatments designed to optimize the microstructural features have been very effective in improving the properties of these materials. However, increasing the overall levels of refractory alloying additions have also negatively impacted the long-term microstructural stability of the alloy and led to issues associated with commercial manufacturing ${ }^{[7-17]}$. Consequently, an improved fundamental understanding of the subtle elemental interactions occurring within these complex engineering alloys must be developed in order to provide resistance to high temperature deformation and maintain solidification characteristics amenable for manufacture.

Traditionally, the temperature capability of Ni-base superalloys has been largely dependent upon the intrinsic ability of these alloys to resist creep deformation. Means to optimize the mechanical performance of these materials generally consisted of thermal treatments to engineer the $\gamma$ ' precipitate microstructure and alloying with high levels of refractory elements to provide solid solution strengthening in both the $\gamma$ and $\gamma^{\prime}$ phases. The degree to which these alloys can be strengthened via solid solution strengthening, however, is limited as the overall composition must be balanced in a manner so that other requirements such as oxidation resistance, coating compatibility, solidification characteristics, long-term microstructural phase stability, density and final cost of the component must also be considered. Currently, many of these limitations imposed by the associated design requirements tend to restrict the ability to further optimize the mechanical properties of these critical structural materials using established empirical correlations and large-scale statistical 
design of experiments. New approaches for achieving significant gains in temperature capability must be coupled with an improved fundamental understanding of the various mechanisms associated with deformation of these alloys. Combining this knowledge with powerful thermodynamic, kinetic and multi-scale deformation models is key in identifying opportunities for continued optimization of these Ni-base superalloy materials. A significant proportion of the intrinsic high temperature strength of single crystal Ni-base superalloys can be directly attributed to the properties of the $\gamma$ and $\gamma^{\prime}$ phases along with the nature of the precipitate - matrix interface. Improving our existing knowledge of the characteristic elemental partitioning tendencies and understanding synergistic effects between the various alloying additions will ultimately contribute to the development of predictive physics-based models linking the effects of chemistry, microstructure and properties.

\section{Experimental Materials and Procedure}

For this investigation, a select group of high refractory content single crystal Ni-base superalloys representative of "third" and "fourth" generation alloys were selected to highlight the dependence of properties on chemistry and processing. Compositions of selected "third" and "fourth" generation single crystal alloys investigated during these preliminary studies are listed in Table I. Five pairs of nominally similar alloys with and without additions of $\mathrm{Ru}$ were used to elucidate the long-term microstructural stabilizing effects associated with Ru. Alloy CMSX-10N, SRR300B, SRR300C, SRR300D and RR2100 are high refractory alloys containing no $\mathrm{Ru}$ additions, while CMSX$10 \mathrm{~N}+3 \mathrm{Ru}, \mathrm{SRR} 300 \mathrm{~B}+3 \mathrm{Ru}, \mathrm{SRR} 300 \mathrm{C}+3 \mathrm{Ru} . \mathrm{SRR} 300 \mathrm{D}+3 \mathrm{Ru}$ and RR2101 contain 2 to $3 w t . \% \mathrm{Ru}$. For the UCSX-series of alloys, the levels of constituent alloying additions were carefully controlled to systematically highlight the effects of overall alloy composition on the solidification characteristics and high temperature mechanical properties.

All of the experimental alloys were directionally solidified as cylindrical single crystal bars measuring $12.7 \mathrm{~mm}$ in diameter and
$700 \mathrm{~mm}$ in length or solid blade-shaped components in the Precision Casting Foundry at Rolls-Royce plc, Derby UK. The solid blade UCSX components were etch inspected using a hot nitric bath in the as-cast condition. The cylindrical test bars were solution heat-treated and aged at appropriate temperatures determined via differential thermal analysis (DTA). Residual segregation in the solution-treated microstructures was quantified using a Cameca SX-100 wavelength dispersive spectrometer (WDS) system. Standard metallographic techniques and scanning electron microscopy (SEM) using a JEOL 6340F FEGSEM were used to assess the microstructure of all the alloys in the as-cast condition and at intermediate stages during the heat-treatment.

The long-term microstructural stability of alloys was evaluated via a series of isothermal exposures at temperatures between $900^{\circ} \mathrm{C}$ to $1100^{\circ} \mathrm{C}$ for times ranging from 1 to 2000 hours. Following the isothermal exposures, the microstructures of the experimental alloys were carefully assessed and quantified in the SEM. The occurrence and fraction of TCP phases was documented as a function of time at temperature. Phase extraction techniques, Xray diffraction and selected area diffraction (SAD) using a JEOL 200CX transmission electron microscope (TEM) were used identify the characteristic crystal structures of the TCP phases that were observed in the various alloys. To investigate the interfacial characteristics between the TCP precipitates and the underlying superalloy matrix, high resolution TEM was performed using a FEI Technai F20 FEGTEM.

The fully solutioned and aged alloys were machined into test specimens and evaluated under a range of creep conditions representative of those encountered by turbine blades during service. Both SEM and TEM analyses were performed on interrupted creep specimens to reveal microstructures corresponding to the initial stages of deformation. For the UCSXseries of alloys, the low cycle fatigue properties were evaluated over a range of stresses at $750^{\circ} \mathrm{C}$ under constant stress conditions. Following testing, the deformed microstructures were evaluated under both the SEM and TEM.

Table I: Nominal Compositions of the Experimental Alloys (wt.\%)

\begin{tabular}{|c|c|c|c|c|c|c|c|c|c|c|c|}
\hline Alloy & Ni & Al & $\mathbf{C r}$ & Co & Mo & Ti & Ta & W & Re & Ru & Hf \\
\hline SRR300B & Bal & 5.8 & 4.0 & 8.0 & 2.2 & - & 7.5 & 4.6 & 5.3 & - & 0.1 \\
\hline SRR300B+3Ru & Bal & 5.6 & 3.9 & 7.8 & 2.1 & - & 7.3 & 4.5 & 5.1 & 3.0 & 0.1 \\
\hline SRR300C & Bal & 5.8 & 4.0 & 8.0 & 2.2 & - & 7.5 & 5.8 & 4.1 & - & 0.1 \\
\hline SRR300C+3Ru & Bal & 5.6 & 3.9 & 7.8 & 2.1 & - & 7.3 & 5.6 & 4.0 & 3.0 & 0.1 \\
\hline SRR300D & Bal & 5.8 & 4.0 & 8.0 & 2.2 & - & 7.5 & 5.2 & 4.7 & - & 0.1 \\
\hline SRR300D+3Ru & Bal & 5.6 & 3.9 & 7.8 & 2.1 & - & 7.3 & 5.0 & 4.6 & 3.0 & 0.1 \\
\hline RR2100 & Bal & 6.0 & 2.5 & 12.0 & - & - & 5.5 & 9.0 & 6.4 & - & - \\
\hline RR2101 & Bal & 6.0 & 2.5 & 12.0 & - & - & 5.5 & 9.0 & 6.4 & 2.0 & - \\
\hline UCSX-1 & Bal & 5.5 & 2.6 & 6.0 & 1.5 & - & 8.1 & 7.0 & 6.2 & 2.0 & 0.1 \\
\hline UCSX-2 & Bal & 5.4 & 3.0 & 8.0 & 1.0 & - & 8.0 & 8.0 & 6.5 & 2.0 & 0.1 \\
\hline UCSX-4 & Bal & 5.5 & - & 8.0 & 1.0 & 0.5 & 8.0 & 8.0 & 6.5 & 2.0 & 0.1 \\
\hline UCSX-5 & Bal & 5.5 & 1.5 & 8.0 & 1.0 & 0.5 & 8.0 & 8.0 & 6.5 & 2.0 & 0.1 \\
\hline UCSX-6 & Bal & 6.3 & - & 4.0 & - & - & 6.0 & 8.0 & 6.8 & 3.0 & - \\
\hline UCSX-7 & Bal & 6.0 & 1.5 & 4.0 & 3.0 & - & 6.0 & 8.0 & 6.8 & 3.0 & - \\
\hline UCSX-8 & Bal & 5.7 & 1.5 & 6.0 & 3.0 & - & 8.0 & 6.0 & 6.0 & 3.0 & - \\
\hline CMSX-10N & Bal & 5.9 & 1.7 & 3.1 & 0.5 & 0.1 & 8.5 & 5.5 & 6.8 & - & - \\
\hline CMSX-10N+3Ru & Bal & 5.7 & 1.7 & 3.0 & 0.5 & 0.1 & 8.4 & 5.3 & 6.6 & 3.0 & - \\
\hline
\end{tabular}




\section{Results}

As expected, there was a strong correlation between the level of refractory alloying additions and the occurrence of macroscopic grain defects, such as freckle formation. A number of investigations have clearly shown that solute segregation during dendritic solidification may potentially induce buoyancy driven convective fluid flow and result in the formation of freckle defects. The magnitude of the buoyancy forces is related to both the level and degree of refractory element segregation. To prevent the onset of these convective instabilities, compositional and/or physical changes in the permeability of the dendritic mush have recently been shown to be effective. In general, the tendency to form freckles decreases with increasing levels of Ta and increases with increasing levels of $\mathrm{W}$ and Re. The solid blade-shaped castings of UCSX-6 to 8 were found to exhibit a range of freckling behavior, Figure 1 and Table II. Alloys UCSX-6 and UCSX-7 are nominally similar except for the addition of $1.5 \mathrm{wt} . \%$ $\mathrm{Cr}$ and 3.0 wt.\% Mo to Alloy UCSX-7. Despite containing a lower overall level of refractory elements, the occurrence of freckle defects on the surface of the solid blade-shaped castings was decreased by a factor of two when solidified under constant processing conditions, Table II. Moreover, despite the fact that Alloy UCSX-8 contains levels of refractory alloying additions that are comparable to those found in UCSX-6, the solid blade shaped components are effectively free of freckle defects. a

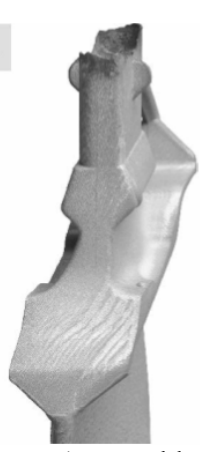

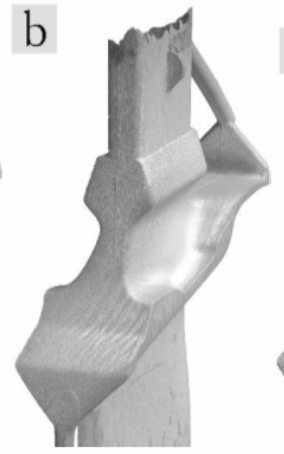

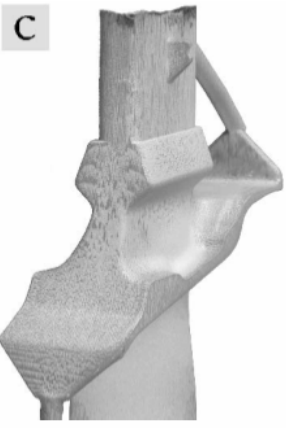

(a) UCSX-6, (b) UCSX-7 and (c) UCSX-8.

Table II: Number and location of defects on the as-cast solid blades

\begin{tabular}{|c|c|c|c|}
\hline Location & UCSX-6 & UCSX-7 & UCSX-8 \\
\hline Tip & $7.5 \pm 1.0$ & 0.0 & 0.0 \\
\hline Blade & $1.5 \pm 0.5$ & 0.0 & 0.0 \\
\hline Platform & $16.3 \pm 1.5$ & $10.8 \pm 1.7$ & $3.0 \pm 1.4$ \\
\hline Root & $18.8 \pm 1.3$ & $9.3 \pm 1.9$ & $0.8 \pm 0.5$ \\
\hline Total Defects & $44.0 \pm 1.4$ & $20.0 \pm 1.2$ & $3.8 \pm 1.3$ \\
\hline
\end{tabular}

Interestingly, the alloying approaches that are utilized to prevent the formation of freckles and misoriented grains were also shown to influence the formation of eutectic scale on the surfaces of the castings. An example can be seen in Figure 2 where two nominally identical alloys, UCSX-6 and UCSX-7 were cast as single crystal turbine blades in a commercial investment casting foundry. Compositionally, these alloys are very similar with the only exception being the additional presence of $1.5 \% \mathrm{Cr}$ and $3.0 \% \mathrm{Mo}$ [wt.\%] in alloy UCSX-7. On the basis of the overall volume fraction of eutectic alone, it is expected that the alloy composition of UCSX-7 should be more resistant to the formation of eutectic scale than UCSX-6 since there is a higher concentration of $\gamma$ forming elements, Mo and $\mathrm{Cr}$, and a lower overall fraction of eutectic in the cast microstructure.

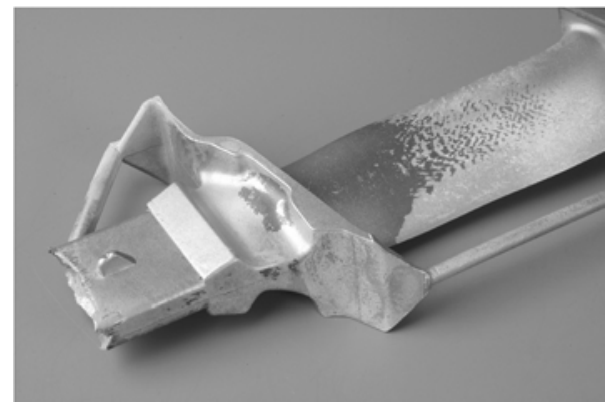

(a)

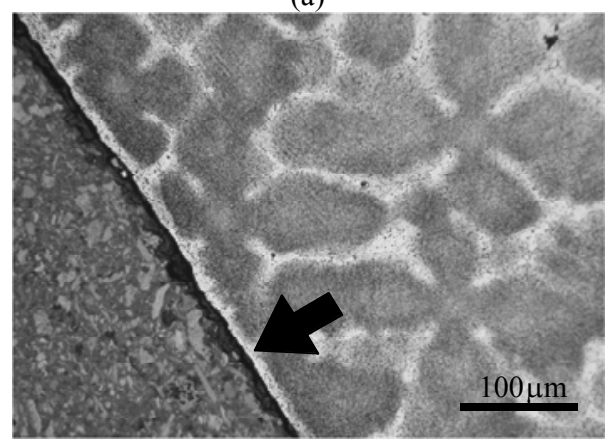

(b)

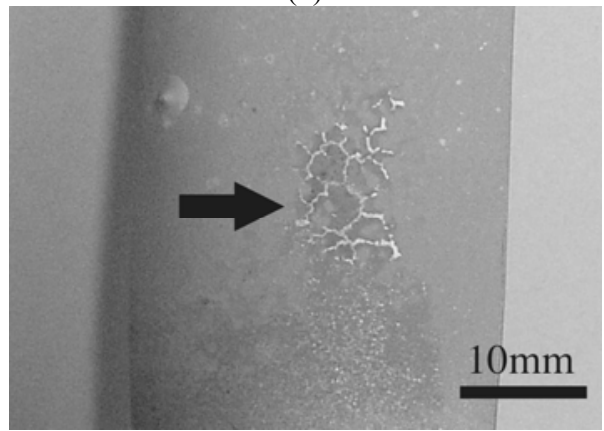

(c)

Figure 2: (a) Eutectic scale on an as-cast single crystal turbine blade. (b) Cross-section showing the presence of a thin coating of eutectic along the surface of a blade. (c) "Wrinkling" of the surface as a result of melting of the eutectic scale during solution heat treatment.

Following an appropriate solution heat treatment and ageing cycle, isothermal exposures at temperatures between $900^{\circ} \mathrm{C}$ and $1100^{\circ} \mathrm{C}$ were used to assess the long-term microstructural stability of the experimental alloys. With the notable exception of CMSX$10 \mathrm{~N}$, all of the non-Ru containing alloys listed in Table I, exhibited a strong tendency form TCP phases after short isothermal exposures. Additions of $\mathrm{Ru}$ to the alloy were observed to effectively suppress and stabilize the microstructure of the experimental high refractory content Ni-base superalloys. Figure 3 shows the corresponding microstructures of alloy SRR300D doped with $0,1.0$ and 3.0wt.\% Ru after a 1000 hour isothermal exposure at $1000^{\circ} \mathrm{C}$. For the alloy containing no $\mathrm{Ru}$, substantial TCP precipitation was observed to occur within the microstructure. Large fractions of refractory-rich $\sigma$ and P-phase precipitates formed throughout the microstructure and destabilized the original $\gamma$ and $\gamma^{\prime}$ structure. The addition of $\mathrm{Ru}$ to the 
SRR300D was found to be particularly effective in preventing the formation of the TCP precipitates after the thermal exposure. A substantially lower fraction of TCP phases were observed in the alloy with the $1.0 \mathrm{wt} . \%$ addition of Ru. When the level of Ru in the alloy was increased to $3.0 \mathrm{wt} . \%$, only localized patches of TCP precipitates were observed within the areas corresponding to the dendrite cores. The stabilizing effects of $\mathrm{Ru}$ additions were found to be consistent over a relatively broad range of compositions as other similar effects were observed when comparing the long-term microstructural stability of SRR300B, SRR300C and RR2100 with their $\mathrm{Ru}$ containing analogues.

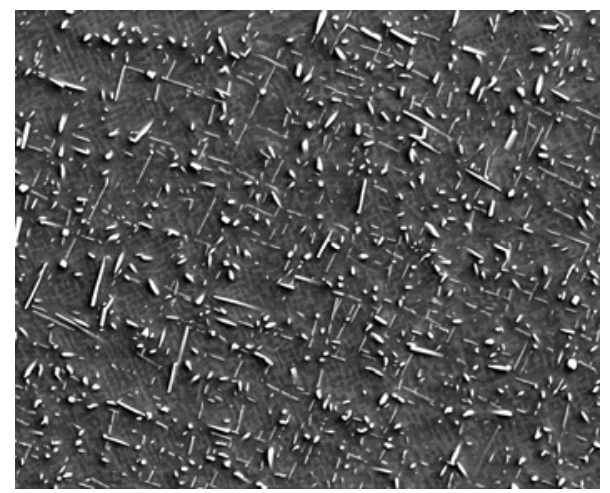

(a)

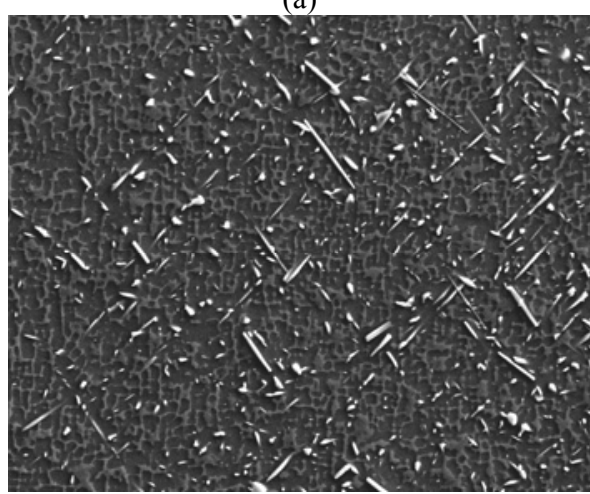

(b)

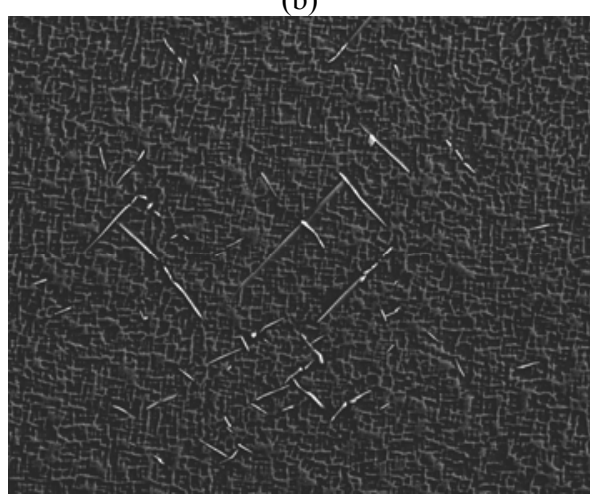

(c)

Figure 2: The presence of TCP precipitates within the microstructure of (a) SRR300D, (b) SRR300+1Ru and (c) SRR300D+3Ru after 1000 hours at $1000^{\circ} \mathrm{C}$.

The long-term microstructural stability of the alloys has a significant impact on the resulting creep capabilities. As precipitation of the TCP progresses, selective leaching of the refractory alloying elements, $\mathrm{Re}, \mathrm{Mo}, \mathrm{Cr}$ and $\mathrm{W}$ occurs and destabilizes the microstructure. This modifies and disrupts the underlying $\gamma-\gamma$ microstructure and leads to a decrease in stress rupture capabilities. Figure 4 shows a comparison of the creep rupture lives of RR2100 and RR2101 at $1100^{\circ} \mathrm{C}$. At comparatively high stresses $(\sim 175 \mathrm{MPa})$, the creep rupture times for both of the alloys are similar at approximately 80 hours. However, as the applied stresses decrease and the corresponding creep rupture times increase, the effect of the $\mathrm{Ru}$ addition becomes more evident as the alloy containing Ru, RR2101, was found to be more resistant to creep deformation. The primary factor contributing to the improvement of creep properties is associated with the stabilization of the microstructure and suppression of the TCP phases. These effects can only be realized after a prolonged exposure to elevated temperatures. In instances where the temperatures and times are insufficient to initiate largescale precipitation of TCP phases, the addition of Ru to the alloy results in a moderate increase in creep resistance.

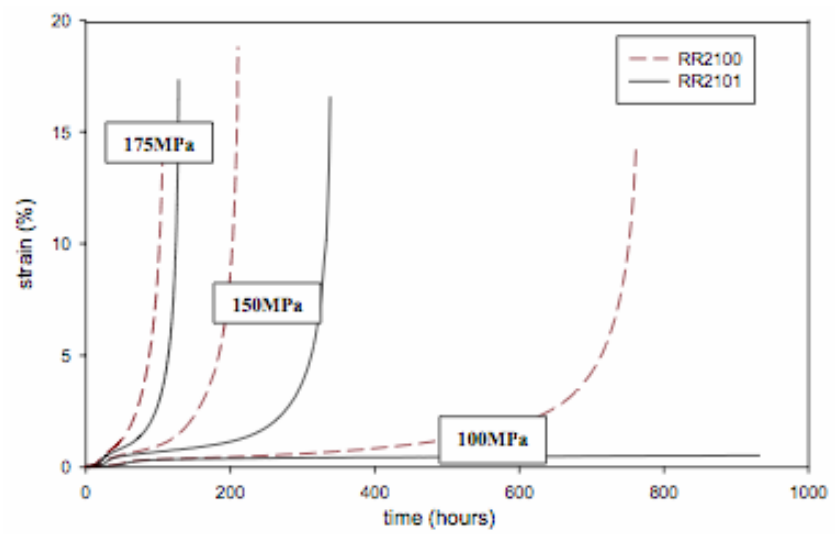

Figure 4: Stress-rupture creep curves of alloy RR2100 and $\mathrm{RR} 2101$ at $1100^{\circ} \mathrm{C}$. The effectiveness of $\mathrm{Ru}$ as a strategic alloying addition increases at lower applied stresses and correspondingly longer time at temperature.

The effect of $\mathrm{Ru}$ on the creep properties of CMSX-10N and SRR300D are compared in a Larson-Miller plot, Figure 5. CMSX-10N possesses a stable microstructure at elevated temperatures, as the extent of TCP formation in the alloy is limited to small amounts. The addition of 3.0wt.\% Ru to CMSX$10 \mathrm{~N}$ does not significantly enhance the creep characteristics of the alloy, particularly at conditions corresponding to low stresses and high temperatures. Only a modest degree of strengthening associated with the $\mathrm{Ru}$ addition was observed under creep at high stresses and low temperatures. For alloys that possess an intrinsically unstable $\gamma-\gamma$ 'microstructure at elevated temperature, the addition of $\mathrm{Ru}$ results in a significant improvement in creep properties. Inhibiting the precipitation and large scale growth of TCP precipitates within the $\gamma-\gamma^{\prime}$ microstructure of alloy SRR300D led to a substantial improvement in creep resistance, particularly at low stress and high temperature test conditions, where a nine-fold increase in stress rupture life was measured. A reasonable improvement in creep properties due to the $\mathrm{Ru}$ addition was evident at low to intermediate temperatures, but the most notable benefits was observed at high temperature, low stress creep conditions. 


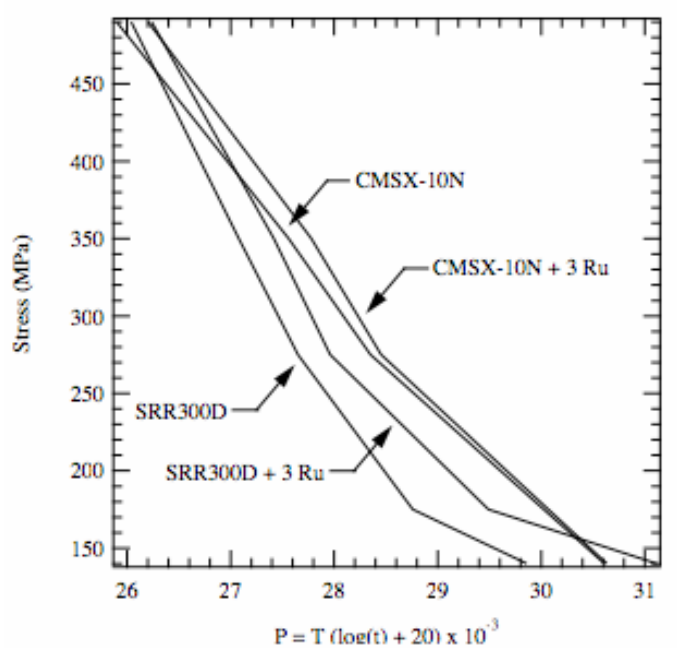

Figure 5: Larson-Miller plot comparing the creep rupture lives of SRR300D, SRR300D+3Ru, CMSX-10N and CMSX-10N+3Ru.

As increasingly high levels of refractory alloying additions are being accommodated into the chemistry of these single crystal alloys, the degree of chemical heterogeneity within the microstructure also tends to increase. Saturation of the $\gamma$ phase with elements possessing limited solubility in the $\gamma^{\prime}$ phase also affects the ability to control the microstructure via heat treatment. Due to the high combined levels of $\mathrm{Cr}$, Mo, $\mathrm{W}$ and Re present in the UCSX-series of alloys, a bi-modal distribution of $\gamma$ ' precipitates was present in the microstructure of the alloys after a standard solution and age heat treatment, Figure 6. Cuboidal $\gamma$ ' precipitates with edge lengths measuring $\sim 400 \mathrm{~nm}$ were observed along with smaller nano-sized cuboidal precipitates contained within the $\gamma$ channels. These fine precipitates were observed to be extremely stable at temperatures below $\sim 870^{\circ} \mathrm{C}$ and did not return into solution after ageing. Due to the stability of these fine $\gamma^{\prime}$ precipitates at temperatures corresponding to low and intermediate temperature creep, their physical presence within the microstructure was observed to influence both the creep and low cycle fatigue properties of the alloy.

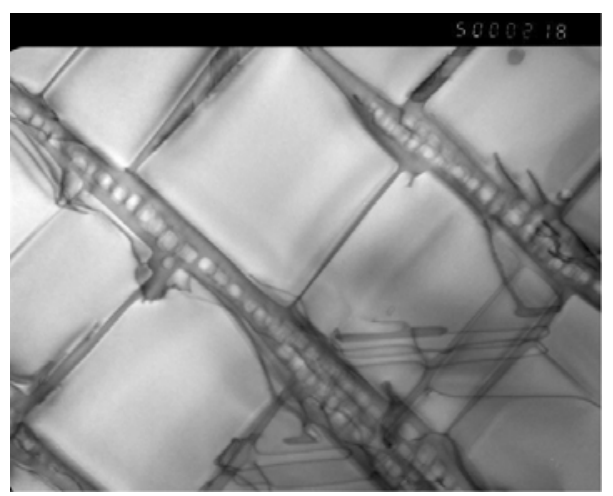

Figure 6: Transmission electron micrograph (TEM) of alloy UCSX-1 after creep deformation at $850^{\circ} \mathrm{C}$. Nano-sized $\gamma^{\prime}$ precipitates are evident within the $\gamma$ channels separating the primary $\gamma^{\prime}$ precipitates.

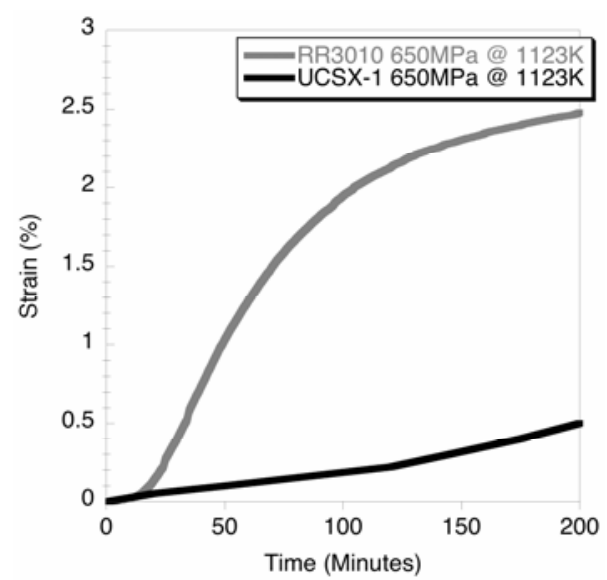

Figure 7: Comparison of the primary creep behavior of a "third" generation single crystal alloy CMSX-10N(RR3010) with a "fourth" generation single crystal alloy UCSX-1 at $1123 \mathrm{~K}$ and $650 \mathrm{MPa}$

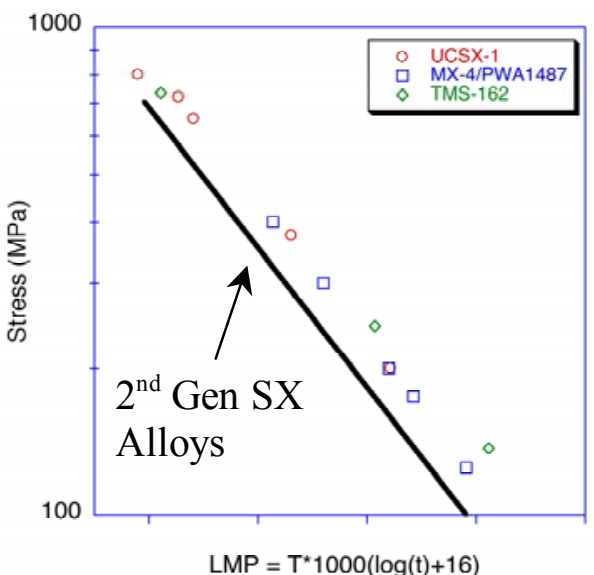

Figure 8: Larson-Miller plot comparing the creep properties of UCSX-1.

Figure 7 shows a comparison of the primary creep behavior of alloy UCSX-1 and RR3010 at $850^{\circ} \mathrm{C} / 650 \mathrm{MPa}$. To minimize anisotropic effects in these single crystal samples, the primary and secondary orientations of both these test specimens were measured and found to be comparable. Although UCSX-1 contains similar overall levels of refractory alloying elements and a lower equilibrium volume fraction of $\gamma^{\prime}$ at these temperatures, the magnitude of the primary creep transient is significantly reduced when compared to RR3010. Under these conditions, the primary creep transient is typically representative $\gamma$ ' precipitate shearing via the activation of $\langle 112>\{111\}$ slip systems. TEM studies on post-mortem creep samples of UCSX-1 did not reveal the presence of artifacts corresponding to $\gamma^{\prime}$ precipitate shearing. Moreover, the unique chemistry of UCSX-1 combined with the novel bi-modal $\gamma^{\prime}$ microstructure was found to significantly enhance not only the low to intermediate temperature creep properties, but the high temperature properties were improved as well. A Larson-Miller plot comparing the creep properties of UCSX-1 with those of the NIMS TMS-162 alloy and the GE/P\&W/NASA MX-4/PWA1497 alloy is shown in Figure 7. The measured creep properties of the UCSX-1 alloys are comparable to these other advanced Ru-bearing Ni-base superalloys and offer significant increases in temperature 
capability when compared to existing second generation single crystal superalloys, such as CMSX-4, ReneN5 and PWA1484.

The resistance of the UCSX-series of alloy to low cycle fatigue (LCF) was also greatly enhanced by the change in chemistry and microstructure. Stress controlled LCF testing conducted at $750^{\circ} \mathrm{C}$ and $20 \mathrm{~Hz}$ showed a large benefit when compared to other second and third generation single crystal Ni-base superalloys. A $\sim 100 \mathrm{MPa}$ improvement in LCF capability over existing single crystal alloys was measured over a range of stresses in UCSX-8. Surprisingly, the LCF capabilities of UCSX-1 were even better than those of UCSX-8 and exhibited a $\sim 150 \mathrm{MPa}$ improvement over the second and third generation single crystal superalloys.

Examination of the deformed LCF specimens in the TEM revealed microstructures atypical of those found in second and third generation single crystals. Only a limited number of dislocation dipoles were evident in the $\gamma$ ' precipitates after LCF deformation at $950 \mathrm{MPa}$. Interestingly, a high density of stacking faults was contained within the narrow $\gamma$ channels.

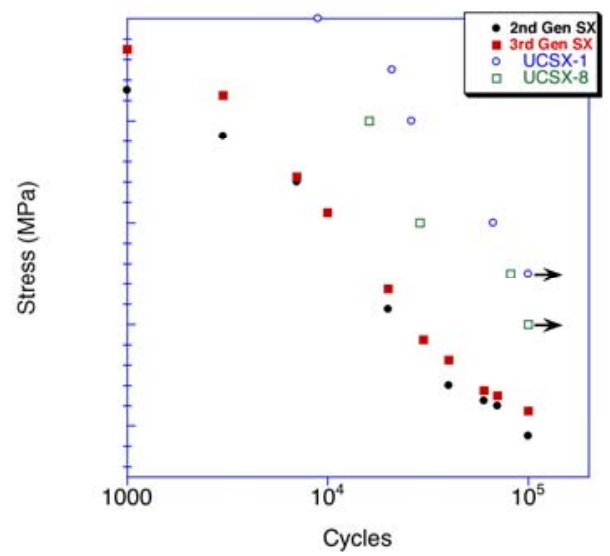

Figure 9: Comparison of low cycle fatigue results from a "second" generation single crystal alloy, a "third" generation single crystal alloy, and UCSX-1 and UCSX-8 "fourth" generation single crystal alloys. UCSX-1 and UCSX-8 exhibit substantially better resistance to $\mathrm{LCF}$ at $750^{\circ} \mathrm{C}$ when compared to existing single crystal superalloys.

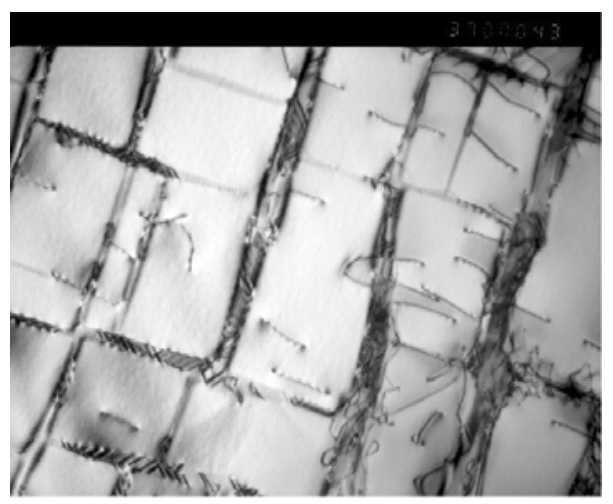

Figure 10: Transmission electron micrograph showing the microstructure of UCSX-1 after low cycle fatigue at $950 \mathrm{MPa}$ and $750^{\circ} \mathrm{C}$. Relatively few dislocation dipoles were present in the $\gamma^{\prime}$ phase, while many stacking faults were observed to reside completely within the narrow $\gamma$ channels.

\section{Discussion}

In order to operate reliably in the hottest sections of the turbine engine, the mechanical properties of high-pressure turbine blades are dependent on the single crystal grain structure. Consequently, control of the as-cast microstructure is of great importance. Although the development of as-cast grain structures has been of interest for many decades, various studies aimed at modeling the formation of solidification structures in a wide range of alloy systems and casting processes have highlighted the complexity associated with simulating the various intrinsic and extrinsic parameters that influence the solidification process ${ }^{[18-25]}$. Moreover, solidification parameters operate over multiple lengthscales, spanning the atomic scale for grain refinement, the micronscale during the formation of the dendritic structure to the macroscopic scale for convective fluid flow phenomena. Thus, knowledge of the underlying mechanisms operating across all of these length scales is required to devise engineering methodologies to minimize the formation of undesirable grain defects during the solidification of single crystal Ni-base superalloy components.

Single crystal Ni-base superalloy castings are typically processed using a Bridgman-type furnace. During this process, control of the solid-liquid interface is maintained by carefully withdrawing a ceramic investment mold through a radiation baffle from the hot zone of a high temperature furnace under vacuum or in an inert gas environment. In commercial furnaces, thermal gradients $(\mathrm{G})$ and withdrawal rates $(\mathrm{R})$ can range from 10 to $100^{\circ} \mathrm{C} / \mathrm{cm}$ and 15 to $50 \mathrm{~cm} / \mathrm{hr}$, respectively. As the size and complexity of the turbine blade designs increase, the limited quantity of radiative heat extraction and the variations in cooling patterns can often lead to the development of macroscopic grain defects and the breakdown of single crystal solidification ${ }^{[11,15,26,27]}$. This holds especially true as the overall content of refractory alloying elements in the alloy increases to enhance strength and extend the temperature capability of the single crystal superalloy. The modest solidification rates combined with severe chemical segregation during solidification leads to large compositional changes between the interdendritic solute within the mushy zone and bulk liquid. Occasionally, the resulting density difference in the liquid triggers localized convective instabilities and initiates plumes of flowing solute that fragment and erode secondary dendrite arms in the mushy zone. This convective flow leads to the breakdown of single crystal solidification as macroscopic grain defects such as freckles and misoriented grains are formed as a result of these instabilities.

Recent investigations have demonstrated the effectiveness of carbon additions in suppressing certain aspects of the mechanisms that lead to freckle formation ${ }^{[28]}$. The physical presence of carbides contained within the mushy zone during solidification combined with subtle changes in the degree of refractory element segregation was demonstrated to effectively suppress the onset of convective instabilities over a wide range of alloy compositions. In the absence of carbon, $\mathrm{Cr}$ and Mo may be used to reduce the extent of $\mathrm{W}$ and Re segregation and mitigate the magnitude of the buoyancy forces driving the localized convective fluid flow ${ }^{[29]}$. Comparing the solidification characteristics of UCSX-6 and UCSX-7 with their chemistries reveals that the inclusion of $1.5 \mathrm{wt} . \% \mathrm{Cr}$ and $3.0 \mathrm{wt} . \%$ Mo greatly reduces the overall number of freckle defects located along the surface of the casting by a factor of two, Figure 1. Quantitative segregation measurements on a wide range of experimental alloys have identified a 
synergistic relationship between $\mathrm{Cr}$, Mo, Re and W during solidification ${ }^{[29]}$. Despite the fact that $\mathrm{Cr}$ and Mo additions should segregate preferentially into the solid during solidification and contribute to the solute density inversion, the resulting synergistic effect on the segregation characteristics of $\mathrm{W}$ and Re are far more beneficial and out weigh the their detrimental effects. Although $\mathrm{Mo}$ and $\mathrm{Cr}$ additions are undesirable with respect to their tendency to induce the precipitation of TCP phases after prolonged exposures to elevated temperatures, these elements also serve to strengthen and enhance the environmental resistance of Ni-base superalloys. Mo is a potent solid solution strengthening addition and $\mathrm{Cr}$ promotes the formation of a protective alumina scale at elevated temperatures.

In addition to the formation of macroscopic grain defects, other undesirable manufacturing anomalies may develop during solidification processing. For particular casting configurations and geometries, the thermal contraction associated with solidification may be constrained by the ceramic investment mold. Thermal contraction during solidification may also play a role in the formation of a eutectic surface scale on the castings. The eutectic surface scale, Figure 2, forms when interdendritic solute is exuded from the mushy zone into the shrinkage gap between the solidified casting and ceramic mold wall. This process is triggered by the gradual changes in pressure associated with the decreasing mass of liquid feeding the casting. Although the presence of the surface scale on the single crystal Ni-base superalloy castings is not structurally damaging, the eutectic phases are prone to incipient melting during the subsequent high temperature solution heat treatments, Figure 2c. Upon resolidification, the eutectic scale takes on the appearance of "surface wrinkles" which impedes the overall manufacturing process since removal requires costly manual reworking of the casting. Based on the compositions of the experimental alloys presented in Table I, the absence of $\mathrm{Cr}$ and Mo from alloy UCSX6 appears to key in the suppression of eutectic scale in the noncarbon containing alloy.

Traditionally, the formation of eutectic surface scale occurs in all non-carbon containing single crystal Ni-base superalloy castings, including CMSX-4, SRR99, PWA1484, etc. The absence of the eutectic scale on UCSX-6 is notable as this alloy contains no carbon or any other minor additions that contribute to the formation of extraneous interdendritic phases. Hence, it is likely that the unique combination of constituent alloying additions is lowering the viscosity of the solute, changing the permeability of the mushy zone or reducing the magnitude of the volume contraction associated with solidification. Furthermore, considering the thickness of the surface scale, it is also possible that this particular combination of alloying elements is influencing the capillarity of the exuded solute and effecting the ability of the solute to fill the narrow shrinkage gap between the solid and the mold wall.

Another alternative method to prevent the formation of eutectic scale on the surface of castings is to utilize minor additions of carbon $^{[30]}$. Carbon additions to Ni-base superalloys were originally intended to strengthen low angle grain boundaries and deoxidize the alloy during vacuum induction melting. However, the presence of carbon also initiates the precipitation of carbides near the dendrite tips during solidification. At sufficiently significant volume fractions, the physical presence of carbides within the mushy zone has been demonstrated to modify the viscosity of the solute and/or the permeability of the mushy zone in a manner that restricts convective fluid flow. These changes are likely to also affect the occurrence of solute exudation during the final stages of solidification. Recently, carbon additions have been found to minimize the occurrence of convective instabilities during single crystal solidification of a broad range of Ni-base superalloy compositions spanning both "second" and "third" generation alloys ${ }^{[28]}$.

Single crystal and directionally solidified components are used exclusively in high temperature applications where resistance to creep deformation is require ${ }^{[31]}$. For single crystal components, structural integrity is required at metal temperatures ranging from $\sim 60-90 \%$ of the melting point, $\mathrm{T}_{\mathrm{M}}$. Depending on the application, the stress states associated with components such as shroud segments, nozzle guide vanes and turbine blades are also highly complex. Turbine engine components are designed such that the load bearing capacity and temperature capability is maximized. Stresses in regions exposed to extremely high temperatures are maintained at comparatively low levels, while high stresses are partitioned to areas where temperatures are comparatively low. Hence, the deformation characteristics of these alloys vary greatly under these conditions and an alloy capable of resisting creep at a wide range of temperatures and stresses is required for these applications. The creep properties exhibited by alloy UCSX-1, Figure 8 , are substantially better throughout the entire temperature range when compared to "second" generation single crystal Nibase superalloys that are commonly in use today. Considering the various creep damage mechanisms that are operative throughout this temperature range, it is notable that the UCSX alloys possess a temperature capability that is $\sim 30$ to $40^{\circ} \mathrm{C}$ higher than the "second" generation alloys.

Comparison of the creep results from SRR300D and SRR300D+3Ru, clearly indicate that the primary benefit of the $R u$ additions is to stabilize the microstructure and suppress TCP precipitation, Figure 5. Stabilization of the microstructure at these temperatures prevents deleterious leeching of the refractory alloying additions that provide solid-solution strengthening from the constituent $\gamma$ and $\gamma$ ' phases as TCP phases form. At lower temperatures where TCP phases thermodynamically unstable or where the diffusion kinetics are too sluggish, the strengthening effect associated with $\mathrm{Ru}$ additions is relatively modest ${ }^{[32]}$. Some improvement in high temperature tensile properties and creep was observed between RR2100 and CMSX-10N, Figures 4 and 5, but the effectiveness of $\mathrm{Ru}$ as an alloying element lies in its ability to restrict the formation of TCP phases at elevated temperatures. This is an extremely important and unique attribute as this effect extends the saturation limit of refractory elements within the $\gamma$ phase and enables the alloy to accommodate higher overall levels of refractory alloying additions to provide high temperature strength or environmental resistance.

In the UCSX-series of alloys, the levels of $\mathrm{Cr}, \mathrm{Mo}, \mathrm{W}$ and $\mathrm{Re}$ additions were systematically increased to take advantage of the stabilizing effect associated with $\mathrm{Ru}$. These are all potent TCPforming elements whose levels are traditionally balanced such that constraints and trade-offs between high temperature strength, environmental resistance and the solidification characteristics commonly exist. The inclusion of $\mathrm{Ru}$ within the alloy extends the limits of alloying with these elements and provides a substantially improved range of flexibility for devising an alloy composition with a balanced combination of properties. With respect to creep 
deformation, the compositional and microstructural changes in the UCSX-series of alloys were responsible for the enhanced creep resistance over a broad range of temperatures from $750^{\circ} \mathrm{C}$ to $1100^{\circ} \mathrm{C}$. At temperatures below $850^{\circ} \mathrm{C}$, the dispersion of nanosized $\gamma$ ' precipitates contained within the narrow $\gamma$ channels were present and impacted the primary creep behavior of the alloy, Figure 6. Only an extremely limited amount of crystal anisotropy resulting from the activation of $<112>\{111\}$ slip systems that lead to accelerated creep deformation was observed, Figure 7 . The resulting changes in composition and microstructure likely impacted both the ability of the $\mathrm{a} / 2<110\rangle$ dislocations to accumulate and dissociate at the $\gamma-\gamma$ ' interfaces. Moreover, the changes in alloy chemistry and partitioning characteristics of the constituent elements will also alter the composition of $\gamma^{\prime}$ phase and possibly lower the anti-phase boundary energy (APB) making the precipitate more resistant to dislocation shearing.

For these high refractory content alloys, creep at intermediate temperatures $\left(\sim 850\right.$ to $\left.950^{\circ} \mathrm{C}\right)$ is highly dependent upon the degree of Orowan strengthening provided by the $\gamma^{\prime}$ precipitates and the intrinsic resistance of the $\gamma^{\prime}$ phase to deformation ${ }^{[33]}$. Typically, the stresses and temperatures characteristics of intermediate temperature creep are insufficient to cause shearing of the $\gamma^{\prime}$ precipitates nor rafting of the cuboidal $\gamma^{\prime}$ precipitates. Additionally, the ability of the nano-sized precipitates contained within the $\gamma$ - channels to provide strengthening is limited as they are likely to have returned into solution at these temperatures. Thus, the large majority of dislocation activity is confined within the narrow $\gamma$ channels during these instances. In order to further restrict the mobility of the dislocations in the $\gamma$ phase, increasingly higher levels of elements such as W, Mo and Re need to be accommodated to lower the stacking fault energy and provide sigh levels of solid solution strengthening. The level of these elements in the alloy, however, is limited by both their tendency to initiate macroscopic grain defects during solidification and to promote the precipitation of TCP phases at elevated temperatures $\left.{ }^{[13,} 17\right]$. Moreover, as the concentration of high-density elements, such as $\mathrm{W}$ and Re increases in the alloy, the overall density of the single crystal blade also increases. This impacts the ability of the blades to be operated at high rotational velocities since the large centrifugal stresses generated by the higher density blades will also require a stronger turbine disk alloy that is capable of retaining the blades under these conditions.

Creep deformation at temperatures above $1000^{\circ} \mathrm{C}$, generally leads to the formation of a "rafted" microstructure ${ }^{[31]}$. Due to the enhanced kinetics that are operative at these temperatures, directional coarsening of cuboidal $\gamma$ ' precipitates occurs and the TCP phases may form if the $\gamma$ phase is supersaturated with $\mathrm{Cr}$, Mo, Re and W. Stabilization of the rafted microstructure against TCP formation was demonstrated to be extremely effective in extending the creep capabilities of the alloy under these conditions $^{[34]}$, Figures 4, 5 and 8 . Other investigations have demonstrated that the degree of interfacial misfit between the $\gamma$ and $\gamma$ ' phases may also influence the creep properties of the alloy under high temperature and low stress conditions. Although the strengthening effects of the lattice misfit may be significant ${ }^{[5]}$, their effectiveness is also dependent upon stabilization of the microstructure against TCP formation ${ }^{[34]}$. The level of refractory alloying additions added to maximize intermediate temperature creep strength needs to be carefully considered since this may potentially degrade the high temperature creep properties. The equilibrium volume fraction of $\gamma^{\prime}$ at elevated temperatures also needs to be carefully controlled since an excessively large fraction of $\gamma^{\prime}$ precipitates may promote supersaturation of the $\gamma$ phase or lead to topological inversion of the matrix phase.

One of the other life limiting issues associated with single crystal turbine blade components is low cycle fatigue (LCF). This type of damage is generally associated with the high cyclic stresses that develop at the root of the single crystal turbine blade where it is mechanically attached to the polycrystalline superalloy rotor disk. Each time the engine is operated, the root section of the blade is required to sustain a stress that is proportional to the rotational velocity of the turbine. With temperatures at the root section of the turbine blade typically being less than $750^{\circ} \mathrm{C}$, the dominant deformation mechanisms responsible for low cycle fatigue damage are also highly sensitive to the intrinsic anisotropy of the single crystal ${ }^{[35]}$. Even modest deviations in crystal orientation may drastically affect the low cycle fatigue behavior of the component. Studies on second generation single crystal Ni-base superalloys have indicated that the onset of crack initiation and growth corresponds to damage accumulation within both phases of the microstructure, as dislocations are able to readily penetrate the $\gamma$ 'precipitates ${ }^{[36,37]}$. Although stacking faults residing within the $\gamma^{\prime}$ precipitates are typically observed within the microstructures of both LCF and creep specimens deformed at similar temperatures, the cyclic stress state associated with fatigue loading also contributes to the formation of dislocation dipole pairs that shear the precipitates.

For these advanced high refractory single crystal alloys containing $\mathrm{Ru}$, however, the characteristic deformation structures after LCF are notably different, Figure 10. Only a limited number of dislocation dipoles are evident in the $\gamma^{\prime}$ precipitates while stacking faults are contained within the narrow $\gamma$ channels. The formation of stacking faults within the narrow $\gamma$ channels has been observed in other heavily alloyed single crystal compositions following low temperature, high stress creep conditions ${ }^{[38]}$. The high concentration of refractory alloying additions in the $\gamma$ phase lowers the stacking fault energy and promotes the presence of widely distended $a / 2<110>$ matrix dislocations during cyclic deformation. Dissociation of these $\mathrm{a} / 2<110>$ matrix dislocations under an applied stress results leads to their formation in the matrix. Moreover, the lack of dislocation activity within the $\gamma$ ' precipitates suggests that the level of alloying within the $\gamma^{\prime}$ precipitates has also increased such that the APB energies may also be correspondingly higher and resistant to dislocation shear. The compositional changes of the $\gamma$ and $\gamma$ ' phases combined with the presence of the nano-sized $\gamma^{\prime}$ precipitates residing within the narrow $\gamma$ channels are all likely contributing to the significant improvement in LCF behavior at $750^{\circ} \mathrm{C}$.

During the past two decades, various experiments have been directed at understanding the characteristic mechanisms by which single crystal Ni-base superalloys accommodate deformation. These investigations have contributed to the development of computational numerical models and various life prediction methodologies that emphasize the importance of improving our fundamental understanding of how these novel materials deform under various temperature and stress combinations ${ }^{[39]}$. Incremental advances in knowledge have corresponded to the relatively minor changes in alloy chemistry differentiating the initial series, or "first generation" of single crystal alloys from the 
"second generation" of alloys that are currently used in all modern turbine engines. However, detailed knowledge of how deformation mechanisms are altered by compositional changes associated with advanced high refractory content or "third generation" and "fourth generation" (high refractory content with platinum group metal additions) single crystal Ni-base superalloys is noticeably lacking.

In summary, single crystal Ni-base superalloys are highly engineered materials that are required to exhibit a wide range of physical and mechanical properties in order to meet the stringent demands required for use as high-pressure turbine blades. In addition to possessing a desirable combination of properties that enable these materials to be resistant to high temperature creep, fatigue, oxidation and sulfidation, these materials need to be amenable for large scale commercial manufacturing processes that include investment casting and heat treatment. As increasing levels of refractory alloying additions are being added to these alloys to improve their mechanical properties and extend their temperature capability, the resulting impact of these compositional changes also influences other characteristics of the alloy, such as the solidification characteristics or the response of the microstructure to heat treatment. In order to meet the needs for improved alloys a detailed understanding of the synergistic dependencies between chemistry, microstructure and processing is required.

\section{Conclusions}

1. Both micro- and macro-alloying approaches may be employed to modify the solidification characteristics of high refractory content Ni-base superalloys and suppress their tendency to form grain defects or scale during casting.

2. Ru additions are particularly effective in suppressing the precipitation and growth of TCP phases in single crystal Ni-base superalloys. The permits higher levels of $\mathrm{W}$, $\mathrm{Re}, \mathrm{Mo}$ and $\mathrm{Cr}$ to be accommodated within the alloy to enhance the physical and mechanical properties of the alloy without destabilizing the microstructure with respect to TCP formation.

3. The strengthening effects associated with $\mathrm{Ru}$ are relatively modest at elevated temperature creep resistance is governed by microstructural stability.

4. Both the low temperature creep behavior and LCF lives are influenced by the presence of a dispersion of nanosized $\gamma$ ' precipitates contained within the $\gamma$ channels.

5. Development of an advanced single crystal alloy requires a number of trade-offs in terms of balancing the need for improved high temperature properties with those of alloy density, cost and manufacturing. Understanding the fundamental mechanisms controlling their characteristic behavior and linking the properties, chemistry, microstructure and processing is necessary for designing alloys for advanced engine applications.

\section{References}

1. Schafrik, R. and Sprague, R., Advanced Materials \& Processes., (2004). Vol. 162. p. 27-30.

2. Walston, W.S., O'Hara, K.S., Ross, E.W., Pollock, T.M., and Murphy, W.H. in Superalloys 1996. (1996). Warrendale, PA: TMS. p. 27-34.
3. Caron, P. in Superalloys 2000. (2000). Warrendale, PA: TMS. p. 737-746.

4. $\quad$ Erickson, G.L. in Superalloys 1996. (1996). Warrendale, PA: TMS. p. 35-44.

5. Zhang, J.X., Murakamo, T., Koizumi, Y., Kobayashi, T., and Harada, H., Acta. Materialia, (2003). Vol. 51. p. 5073-5081.

6. Walston, W.S., Cetel, A., MacKay, R., O'Hara, K., Duhl, D., and Dreshfield, R. in Superalloys 2004. (2004). Warrendale, PA: TMS. p. 15-24.

7. Wlodek, S.T. in Long Term Stability of High Temperature Materials. (1999). Warrendale, PA: TMS. p. $1-38$.

8. Yeh, A.C., Rae, C.M.F., and Tin, S. in Superalloys. (2004). Warrendale, PA: TMS. p. 677-686.

9. Yeh, A.C. and Tin, S. in Parsons 2003. (2003): IOM. p. 673-686.

10. Tin, S. and Pollock, T.M., Journal of Materials Science, (2004). Vol. 39. p. 7199-7205.

11. Tin, S., Pollock, T.M., and Murphy, W., Metall. Mater. Trans., (2001). Vol. 32A. p. 1743-1753.

12. Rae, C.M.F., Karunaratne, M.S.A., Small, C.J., Broomfield, R.W., Jones, C.N., and Reed, R.C. in Superalloys 2000. (2000). Warrendale, PA: TMS. p. 767-776.

13. Rae, C.M.F. and Reed, R.C., Acta Materialia, (2001). Vol. 49. p. 4113-4125.

14. Pollock, T.M., Murphy, W.H., Goldman, E.H., Uram, D.L., and Tu, J.S. in Superalloys 1992. (1992).

Warrendale, PA: TMS. p. 125-134.

15. Pollock, T.M. and Murphy, W.H., Metall. Mater. Trans., (1996). Vol. 27A. p. 1081-1094.

16. Nystrom, J.D., Pollock, T.M., Murphy, W.D., and Garg, A., Metall. and Mater. Trans., (1997). Vol. 28A. p. 2443-2452.

17. Darolia, R., Lahrman, D.F., and Field, R.D. in Superalloys 1988. (1988). Warrendale, PA: TMS. p. 255-264.

18. Beckermann, C., Gu, J.P., and Boettinger, W.J., Metall. Mater. Trans., (2000). Vol. 31A. p. 2545-2557.

19. Frueh, C., Poirier, D.R., and Felielli, S.D., Metall. Mater. Trans., (2000). Vol. 31A. p. 3129-3135.

20. Gu, J.P., Beckermann, C., and Giamei, A.F., Metall. and Mat. Trans., (1997). Vol. 28A. p. 1533-1542.

21. Saunders, N., Fahrmann, M., and Small, C.J. in Superalloys 2000. (2000). Warrendale, PA: TMS. p. 803-811.

22. Schneider, M.C. and Beckermann, C., International Journal of Heat and Mass Transfer, (1995). Vol. 38. p. 3455-3473.

23. Schneider, M.C., Gu, J.P., Beckermann, C., Boettinger, W.J., and Kattner, U.R., Metall. Trans., (1997). Vol. 28A. p. 1517-1531.

24. Yu, K.O., Nichols, J.J., and Robinson, M., JOM, (1992). Vol. 44. p. 21-25.

25. Yu, K.O., Oti, J.A., Robinson, M., and Carlson, R.G. in Superalloys 1992. (1992). Warrendale, PA: TMS. p. 134-144.

26. Copley, S.M., Giamei, A.F., Johnson, S.M., and Hornbecker, M.F., Metall. Trans., (1970). Vol. 1. p. 2193-2204.

27. Giamei, A.F. and Kear, B.H., Metall. Trans., (1970). Vol. 1. p. 2185-2192. 
28. Tin, S. and Pollock, T.M., Metal. Mater. Trans. A, (2002). Vol. 34. p. 1953-1967.

29. Hobbs, R.A., Tin, S., and Rae, C.M.F., Metall. Mater Trans. A, (2005). Vol. 36A. p. 2761-2773.

30. Milhalisin, J.R., Corrigan, J., Launsbach, M., Leonard, E., Baker, R., and Griffin, B. in Superalloys 2004.

(2004). Warrendale, PA: TMS. p. 795-800.

31. Pollock, T.M. and Tin, S., J. Power and Propulsion, (2006). Vol. 22. p. 361-375.

32. Yeh, A.C. and Tin, S., Scripta. Mater., (2005). Vol. 52. p. 519-524.

33. Pollock, T.M. and Argon, A.S., Acta. Metall. Mater., (1992). Vol. 40. p. 1-30.

34. Yeh, A.C. and Rae, C.M.F. in Superalloys 2004. (2004). Warrendale, PA: TMS. p. 677-686.

35. Arakere, N.K. and Swanson, G., Journal of Engineering for Gas Turbines and Power, (2002). Vol. 124. p. 161176.

36. Clavel, M. and Pineau, A., Mater. Sci. Eng., (1982). Vol. 55. p. 157-171.

37. Chan, K.S., Hack, J.E., and Leverant, G.R., Metall. Trans. A, (1987). Vol. 18A. p. 581-591.

38. Ma, S., Carroll, L., and Pollock, T.M., Acta Materialia, (2007). Vol. 55. p. 5802-5812.

39. Basoalto, H.C., Ghosh, R.N., Ardakani, M.G., Shollock, B.A., and McLean, M. in Superalloys. (2000).

Warrendale, PA. p. 515-524. 